\title{
Limited resection trial for pulmonary ground-glass opacity nodules: Fifty-case experience
}

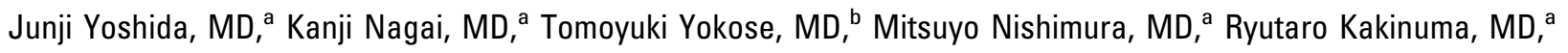
Hironobu Ohmatsu, MD, and Yutaka Nishiwaki, MDa

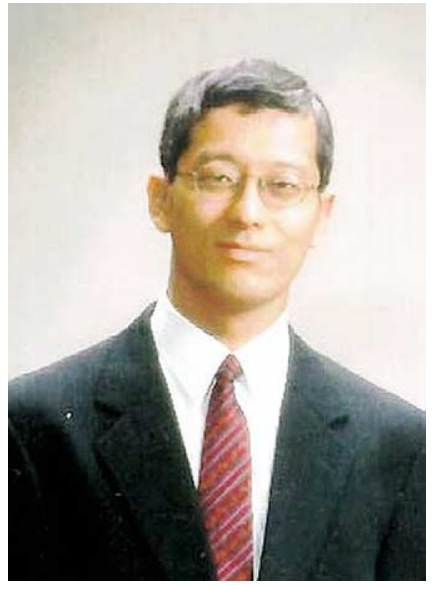

Dr Yoshida
From the Department of Thoracic Oncology, National Cancer Center Hospital East, ${ }^{\mathrm{a}}$ and the Pathology Division, National Cancer Center Research Institute East, ${ }^{\mathrm{b}}$ Kashiwa, Japan.

Supported in part by a Grant-in-Aid for Cancer Research from the Ministry of Health, Labor and Welfare, Japan.

Received for publication Dec 28, 2002; revisions received June 25, 2004; accepted for publication July 21, 2004.

Address for reprints: Junji Yoshida, MD, Department of Thoracic Oncology, National Cancer Center Hospital East, 6-5-1, Kashiwanoha, Kashiwa, Chiba, 277-8577, Japan (E-mail: jyoshida@east.ncc.go.jp).

J Thorac Cardiovasc Surg 2005;129:991-6 $0022-5223 / \$ 30.00$

Copyright () 2005 by The American Association for Thoracic Surgery

doi:10.1016/j.jtcvs.2004.07.038
Objective: This study was undertaken to determine the recurrence rate after limited resection of small lung carcinoma and to evaluate intraoperative frozen-section examination accuracy for Noguchi classification.

Methods: Enrollment requirements were as follows: pulmonary nodule $2 \mathrm{~cm}$ or smaller, diagnosed or suspected clinical T1 N0 M0 carcinoma in the lung periphery, and ground-glass opacity findings and lack of evident pleural indentations or vascular convergence on high-resolution computed tomographic scan. A wedge or segmental resection specimen, removed with custom stapler cartridges, was immediately reinflated and examined by frozen-section with hematoxylin-eosin and Victoria blue-van Gieson stains. If the tumor was confirmed as Noguchi type A or $B$ with resection margins greater than $1 \mathrm{~cm}$, the patient was closed and followed up on an outpatient basis. End points were 5-year disease-free survival and intraoperative classification accuracy.

Results: From August 1998 through October 2002, a total of 50 patients were enrolled (20 men and 30 women, ages 30-77 years). Tumor sizes ranged from 2 to $21 \mathrm{~mm}$ (11 mm average). There were 2 Noguchi type A tumors, 23 Noguchi type B tumors, 15 Noguchi type $\mathrm{C}$ tumors, 5 atypical adenomatous hyperplasias, 4 fibroses, and 1 granuloma. Frozen-section accuracy was approximately 98\% (39/ 40). One intraoperative type B diagnosis was revised to type $\mathrm{C}$ after postoperative pathologic study. No morbidity, mortality, or recurrence has been seen with a median follow-up of 50 months.

Conclusion: Noguchi type A and B tumors may well be in situ carcinomas, and frozen-section examination was highly accurate. Neither local recurrence nor distant metastases have been found to date. Limited resection initial results appear promising.

$\mathrm{T}$ he Lung Cancer Study Group has performed the only prospective, randomized trial of limited resection versus lobectomy to date. They concluded that lobectomy was the appropriate surgical treatment for T1 N0 M0 non-small cell lung carcinomas, because limited resection resulted in greater local recurrence. ${ }^{1}$ The Lung Cancer Study Group trial did not include many small cancers, and ground-glass opacity $(\mathrm{GGO})^{2}$ nodules were not recognized at the time of the study. Since then, several researchers have reported, although in retrospective study designs, that limited resection could be an acceptable alternative for patients with $\mathrm{T} 1$ N0 M0 disease. ${ }^{3,4}$ We decided to do more investigations and evaluations. In 1998, we reviewed peripheral lung cancers smaller than $1 \mathrm{~cm}$ in diameter and found that almost half of them displayed an invasive nature. We concluded that tumor size alone cannot be a positive indicator for limited resection. ${ }^{5}$

Shimosato and colleagues ${ }^{6}$ retrospectively evaluated cancer fibrotic focus or scarring and patient prognosis. They found that increasing fibrotic focus or scarring 


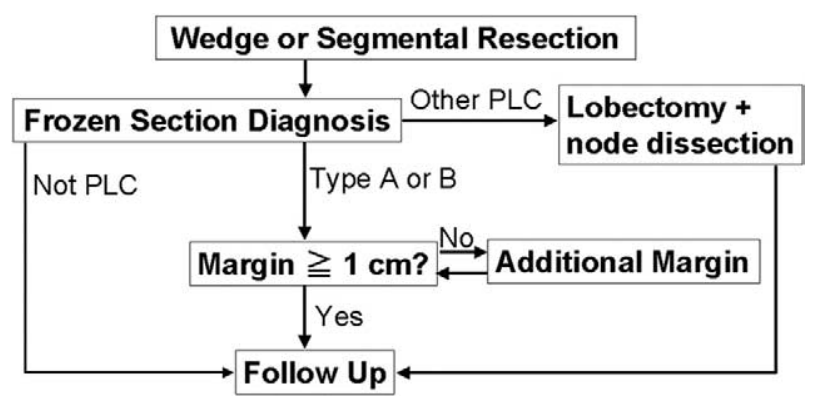

Figure 1. Treatment sequence. PLC, Primary lung cancer

was positively related to pleural invasion, lymph node metastasis, and blood vessel invasion. Patient prognosis was poorer with increased focus or scarring. They believed that scarring and resulting pleural indentations occurred with tumor development.

Noguchi and associates ${ }^{7}$ developed a six-category classification for lung adenocarcinomas less than $2 \mathrm{~cm}$ in diameter. They concluded, on the basis of histologic characteristics and outcomes, that localized bronchioloalveolar carcinoma (type A) and localized bronchioloalveolar carcinoma with foci of collapse (type B) were pathologically and biologically in situ noninvasive peripheral carcinomas, whereas localized bronchioloalveolar carcinoma with foci of fibroblastic proliferation (type $\mathrm{C}$ ) was an advanced invasive stage of types $\mathrm{A}$ and $\mathrm{B}$. This differentiation required careful histologic examination.

We speculated that if Noguchi type A and B tumors truly are in situ, noninvasive carcinomas, limited resection might be the procedure of choice. With this goal in mind, we developed some unique tools and methods for intraoperative diagnosis of these tumor types. With these methods, we found that we were able to reliably differentiate Noguchi types $\mathrm{A}$ and $\mathrm{B}$ from type $\mathrm{C}$ during the surgical procedure. As a result, in 1998 at our institution we started a prospective limited resection clinical trial to confirm the reliability

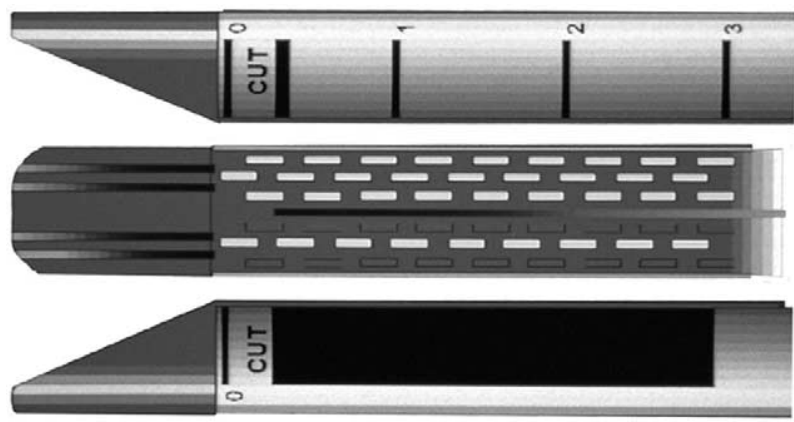

Figure 2. Customized stapler (ENDO-GIA 30 3.5/3-1; Tyco Healthcare Japan, Tokyo, Japan). of the tumor type differentiation methods and to determine the survival of those with limited resection based on the tumor differentiation results for probable in situ adenocarcinoma in the lung periphery. We describe the study design, unique tools and methods, and preliminary results after completion of the planned 50 patient enrollment.

\section{Patients and Methods}

Our objectives were to evaluate intraoperative frozen-section examination in the identification of Noguchi type A or B tumors, to perform limited resection for Noguchi A and B and other benign or noninvasive nodules, and to determine the extent of local recurrence or distant metastases in all patients at 5-year follow-up. Enrollment required patients with a tumor less than $2 \mathrm{~cm}$ in diameter diagnosed or suspected as a clinical T1 N0 M0 carcinoma in the lung periphery on the basis of a computed tomographic (CT) scan. They had to have a high-resolution CT scan with findings suggestive of a Noguchi type A or B tumor: GGO and lack of evident pleural indentations or vascular convergencWritten informed consent was obtained from each participant. Patients with a malignancy history within the past 5 years and those not candidates for lobectomy and systematic lymph node dissection were excluded.

Figure 1 shows the treatment flow chart. We performed wedge or segmental resection, depending on the tumor location. When a tumor was deep in the middle of a segment, segmentectomy was chosen. Also, when a tumor could not be localized during surgery, we performed a segmentectomy to avoid missing the tumor. Our pathologist (T.Y.) examined the frozen-section specimen immediately. If the tumor was confirmed as Noguchi type A or B with a resection margin greater than $1 \mathrm{~cm}$, the patient was closed up and followed up on an outpatient basis. If the margin was not sufficient, additional margin was resected. If the tumor was a primary malignancy, but not type A or B, lobectomy and systematic lymph node dissection were performed. Patients are followed up on an outpatient basis at least every 6 months by physical check-up, plain chest radiograph, and laboratory tests. Patients who underwent limited resection for Noguchi type A or B disease have chest CTs every year.

It is fairly difficult to perform Noguchi classification from a simple frozen section. To facilitate the examination, we used several innovative tools and techniques in our trial. We believe these contributed significantly to the trial results. Figure 2 shows the stapler cartridge (ENDO-GIA 30 3.5/3-1; Tyco Healthcare Japan, Tokyo, Japan), custom modified as requested by our chief thoracic surgeon (K.N.). Rather than three lines of staples on both sides, it has a single staple line on the black, resected specimen side. This makes it easier to cut a narrow pathologic examination specimen margin strip for negative cut-end confirmation.

With no obvious specimen bronchus or bronchiole for phosphate-buffered saline solution injection, specimen inflation to facilitate alveolar structure observation is difficult. To inflate the resected specimen's alveolar structure, our pathologist used a technique known, but not normally used in neoplastic lung disease diagnosis. ${ }^{9}$ With the specimen in a closed, phosphate-buffered saline solution-filled syringe, the piston was pulled back quickly and repeatedly, reinflating the alveolar structure by replacing alveolar air with phosphate-buffered saline solution (Figure 3). After 


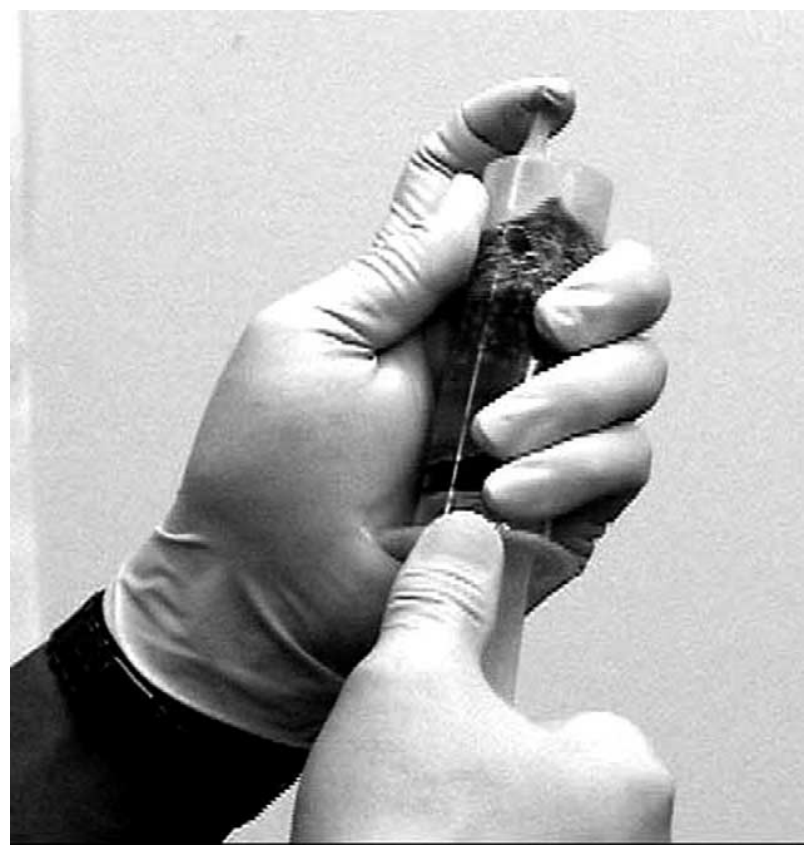

Figure 3. Specimen vacuum reinflation.

slicing the specimen into 2-mm thick slices and a second reinflation, the pathologist stereoscopically examined the slices, looking for the most severe structure destruction indications. The maximum stromal destruction slices or the largest area slice were cut by cryostat, stained with hematoxylin-eosin, and examined microscopically.

In addition to routine hematoxylin-eosin staining, our pathologist (T.Y.) thought Victoria blue-van Gieson (VvG) staining would improve Noguchi classification accuracy, because it reveals whether the alveolar wall elastic fibers are intact, ${ }^{10}$ providing a powerful aid in improving intraoperative classification accuracy. If the elastic fibers were destroyed by tumor cells, as shown in Figure $4, A$, the tumor was diagnosed as Noguchi type $\mathrm{C}$, whereas if they were intact, as in Figure 4, B, the classification was type A or B. This diagnostic judgment was based on our group's previous report (Yokose and associates ${ }^{11}$ ) that patients without stromal destruction, as shown by $\mathrm{VvG}$ staining, survived 5 years without recurrence.

Noguchi and associates ${ }^{7}$ reported no cancer recurrences at 5 years after lobectomy and lymph node dissection in the type A and B population. ${ }^{7}$ Statistically, it is impossible to compare the limited resection outcome for these tumors with the event-free standard surgery outcome. We reported an $85 \%$ 5-year survival rate among patients with tumors $2 \mathrm{~cm}$ or smaller and T1 N0 M0 pathologic class who underwent lobectomy and systematic lymph node dissection at our institution. ${ }^{12}$ Allowing 4 local recurrences in 50 patients, a $90 \%$ confidence interval of $86 \%$ to $98 \%$ results, and the lower limit would be better than our earlier study. We therefore decided to recruit 50 patients, with a trial-quitting rule of 5 local recurrence cases.

The first end point was accurate frozen-section examination results for carcinoma invasiveness. The second, and more impor-
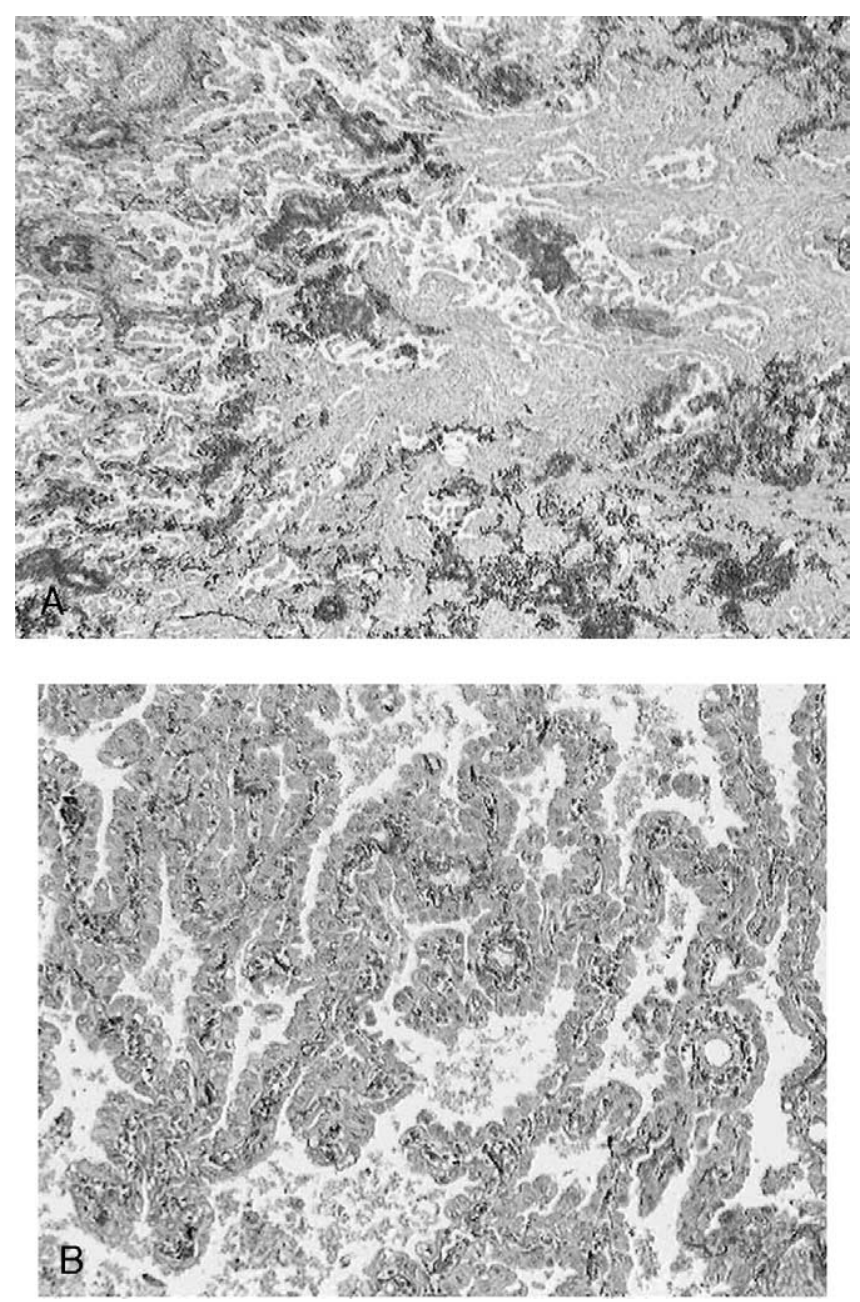

Figure 4. VvG staining showing elastic fiber destruction in type C tumor $(A)$ and intact elastic fibers in type $A$ tumor (B). (Magnification $100 \times$.)

tant, end point was 5-year disease-free survival. This study protocol was reviewed by the National Cancer Center Hospital East institutional review board and was approved in July 1998.

\section{Results}

This prospective study (August 1998-October 2002) enrolled 50 patients comprising only $5.3 \%$ of all patients with resected lung cancer at our institution during this period. There were 20 men and 30 women, with ages ranging from 30 to 77 years (average 61 years). Tumor sizes ranged from 2 to $21 \mathrm{~mm}$ (average $11 \mathrm{~mm}$ ). Thirty patients had wedge resection, 6 segmentectomy, and 14 lobectomy with lymph node dissection. Nineteen of the 30 wedge procedures were performed thoracoscopically by three-port access, and the other 11 needed a small thoracotomy.

Three-port procedures were performed when the tumor was subpleural or when the tumor was shallow or hard 
TABLE 1. Histologic subtype and tumor size distribution in resected specimens

\begin{tabular}{lcccc}
\hline Subtype & $\begin{array}{c}\text { No. of } \\
\text { cases }\end{array}$ & $\begin{array}{c}\text { Range } \\
(\mathbf{m m})\end{array}$ & $\begin{array}{c}\text { Median } \\
\text { (mm) }\end{array}$ & $\begin{array}{c}\text { Resection } \\
\text { type } \\
\text { (W/S/L) }\end{array}$ \\
\hline Type A & 2 & $9-10$ & - & $1 / 1 / 0$ \\
Type B & 23 & $6-21$ & 12 & $21 / 2 / 0$ \\
Type C & 15 & $10-19$ & 14 & $1 / 0 / 14$ \\
$\begin{array}{l}\text { Atypical adenomatous } \\
\quad \text { hyperplasia }\end{array}$ & 5 & $5-14$ & 8 & $2 / 3 / 0$ \\
$\begin{array}{l}\text { Fibrosis } \\
\text { Granuloma }\end{array}$ & 4 & $6-15$ & 10 & $4 / 0 / 0$ \\
\hline
\end{tabular}

$W / S / L$, Wedge resection/segmentectomy/lobectomy plus lymph node dissection, in numbers of patients.

enough to directly palpate with one or two fingers through the ports. Initially, because of the nature of GGOs, we were not sure that we would be able to palpate and localize these lesions. However, we found the GGO-containing lung parenchyma to have a different texture than the surrounding normal parenchyma. When it was impossible to determine the tumor location or periphery through the thoracoscopy ports, the procedure was converted to a small thoracotomy. Segmentectomy and lobectomy were done through a musclesparing thoracotomy, typically about $12 \mathrm{~cm}$ in length.

During the trial, Noguchi classification assessment took about an hour because of the extensive image recording required for study purposes. There were 2 Noguchi type A tumors, 23 Noguchi type B tumors, 15 Noguchi type C tumors, 5 atypical adenomatous hyperplasias (AAH), 4 fibroses and 1 granuloma. Their size distribution is summarized in Table 1. In addition to the intraoperative slides, further postoperative slides were prepared and studied. The postoperative slides did not differ significantly from the intraoperative slides. One initial frozen-section type B diagnosis was revised to type $\mathrm{C}$ after postoperative pathologic study. We discussed this with the patient in detail, and he chose not to have any further treatment. He is still alive without any signs of recurrence after more than 5 years.

No morbidity or mortality has been seen. During the follow-up period, with a range of 19 to 68 months (median 50 months), as this is being written (May 2004), there have been no recurrences. Enrollment concluded without a forced quit.

\section{Discussion}

The Lung Cancer Study Group conducted a prospective randomized trial to evaluate the role of limited resection versus lobectomy for T1 N0 M0 non-small cell lung carcinomas. They reported significantly increased local recurrence and marginally but not significantly higher cancer death rates in the limited resection group relative to the lobectomy group. On the basis of their observa- tions, they concluded that lobectomy was the surgical treatment of choice for patients with T1 N0 M0 nonsmall cell lung carcinomas. ${ }^{1}$ However, there has been some question whether limited resection is always contraindicated. We reported a retrospective review of peripheral lung cancers of all types less than $1 \mathrm{~cm}$ in diameter in $1998 .^{5}$ Of the 16 small tumors, 7 displayed an invasive nature. We therefore concluded that tumor size alone is not a sufficient indicator for limited resection. This observation is consistent with a recent report on subcentimeter lung cancers from the Mayo Clinic. ${ }^{13}$ However, in patients with impaired respiratory function, limited resection has been tried and has often yielded acceptable outcomes. ${ }^{14}$ Several researchers have reported, although in retrospective studies, that limited resection could be an acceptable alternative in patients with T1 N0 M0 disease and insufficient pulmonary reserve. ${ }^{3,4}$

Noguchi and associates ${ }^{7}$ conjectured that type A and type $B$ tumors are in situ carcinomas, whereas type $C$ is an advanced stage of types A and B. If Noguchi type A and B peripheral tumors are truly in situ, noninvasive carcinomas, limited resection would be the management of choice for these tumors. As a result, in August 1998 we started this prospective clinical trial with intraoperative frozen-section examination to establish the Noguchi classification and limited resection for probable in situ adenocarcinoma with GGO characteristics in the lung periphery.

As noted, Shimosato and associates ${ }^{6}$ evaluated cancer fibrotic focus or scarring and patient prognosis. Increased lymph node metastasis and pleural and blood vessel invasion were present with greater scarring and fibrotic focus. Aoki and colleagues ${ }^{15}$ noted the pleural indentation and vascular convergence increased with tumor development in Noguchi type B and C tumors. This information was incorporated into our patient selection criteria.

A concern in our trial was the accuracy of frozensection examination. The correct classification as atypical adenomatous hyperplasias or Noguchi type $\mathrm{A},{ }^{16}$ or as Noguchi type B or type $\mathrm{C}$ tumors, has been reported as being difficult, even more so with frozen sections. We think that the equipment developed for this trial and the methods and techniques applied contributed significantly to our outcomes. Finding the GGO "spongelike" structure could be felt in the lung made locating the lesion and ensuring sufficient resection margin much easier. The customized stapling cartridge and negative-pressure specimen inflation were useful in frozen-section preparation. They made it much easier to work with the specimen. Stereoscopic microscopy enabled the pathologist to locate regions of interest for detailed examination. For Noguchi subtype determination, $\mathrm{VvG}$ staining proved to be a powerful aid in separating Noguchi type A and B 
from type C. These tools, methods, and techniques, together with the expertise of our pathologist, resulted in high frozen-section examination accuracy: only 1 type B lesion in 50 cases was recategorized as type $\mathrm{C}$ postoperatively. This patient underwent only a wedge resection and, after being fully informed of the underdiagnosis, potential outcomes, and additional treatment options, decided not to undergo any further treatment. He is still alive after more than 5 years without recurrence.

The other patients with 14 Noguchi type C tumor, whose diagnoses were confirmed by postoperative pathologic study, underwent lobectomy and systematic lymph node dissection after the frozen-section diagnosis. Detailed pathologic study after the operation revealed no nodal involvement, pulmonary metastases, lymphatic permeation, or vascular invasion in the specimens. It is likely that these patients will survive. Kondo and colleagues ${ }^{17}$ studied air-containing lesions $2 \mathrm{~cm}$ or smaller, including GGO or subsolid tumors. These lesions were of interest if their opacity area decreased by more than $50 \%$ on the mediastinum-setting CT from the lung-setting CT. They reported that this patient cohort survived 5 years without recurrences after either standard or limited resection, and most of them had no node metastases or vessel involvement. So although Noguchi type $\mathrm{C}$ tumors are invasive, they may well represent an early invasive stage. On the basis of our finding of no nodal involvement, lymphatic permeation, or vascular invasion, and Kondo and colleagues' similar results and survival rates, ${ }^{17}$ we conjecture that Noguchi type C tumors in our trial might well also have been curatively treated by limited resection. However, we did not address this issue in our trial.

During the trial, Noguchi classification assessment took about an hour. This was mostly because we extensively recorded sample images for study purposes. Our pathologist believes that in routine practice, without extensive image recording, the determination can be accomplished in 15 to 20 minutes.

There was no definite difference in size distribution among subtypes. However, all Noguchi type $\mathrm{C}$ tumors were $1 \mathrm{~cm}$ or larger, whereas other subtypes included subcentimeter tumors. This strengthens the suggestion that limited resection is indicated when a GGO tumor is smaller than 1 $\mathrm{cm}$. Although this contradicts our previous review ${ }^{5}$ and a recent report on subcentimeter lung cancers by the Mayo Clinic, ${ }^{13}$ that is probably because those series included non-GGO lesions.

In conclusion, Noguchi classification type A and B tumors appear to be in situ, noninvasive carcinomas, and limited resection achieves the goals of local control and survival. With about 5.5 years total on this study, it may be still too early for strong conclusions. Considering the probable slow-growing nature of GGO lesions, ${ }^{18} 5$ years of follow-up is not long enough to conclude that the disease is cured. We will probably have to continue our follow-up for an additional 5 years. However, initial results are encouraging. With the customized stapling cartridges, negative-pressure specimen preparation and inflation, stereoscopic microscopy, $\mathrm{VvG}$ staining, and a skilled pathologist, frozen-section classification of Noguchi subtype has been highly accurate. Our results suggest that lung tumors $2 \mathrm{~cm}$ or less in diameter with highresolution CT scan findings of GGO and without evident pleural indentations or vascular convergence may be safely managed with only limited resection.

We thank the International Early Lung Cancer Action Program for presentation opportunities and encouraging us to report our results. We are indebted to Professor Joe B. Putnam, Jr, Chair of the Department of Thoracic Surgery of Vanderbilt University Medical Center, Nashville, Tenn, for his manuscript review. The principal author thanks his friend Mr Brian Curry, communication consultant, for his continuous help in focusing on clarity, conciseness, and comprehension.

\section{References}

1. Ginsberg RJ, Rubinstein LV. Randomized trial of lobectomy versus limited resection for T1 N0 non-small cell lung cancer. Lung Cancer Study Group. Ann Thorac Surg. 1995;60:615-22.

2. Austin JH, Muller NL, Friedman PJ, Hansell DM, Naidich DP, RemyJardin M, et al. Glossary of terms for CT of the lungs: recommendations of the Nomenclature Committee of the Fleischner Society. Radiology. 1996;200:327-31.

3. Kodama K, Doi O, Higashiyama M, Yokouchi H. Intentional limited resection for selected patients with T1 N0 M0 non-small cell lung cancer: a single-institution study. J Thorac Cardiovasc Surg. 1997; 114:347-53.

4. Pastorino U, Valente M, Bedini V, Infante M, Tavecchio L, Ravasi G. Limited resection for stage I lung cancer. Eur J Surg Oncol. 1991;17: 42-6.

5. Yoshida J, Nagai K, Yokose T, Takahashi K, Nishimura M, Goto K, et al. Primary peripheral lung carcinoma smaller than $1 \mathrm{~cm}$ in diameter. Chest. 1998;114:710-2.

6. Shimosato Y, Suzuki A, Hashimoto T, Nishiwaki Y, Kodama T, Yoneyama T, et al. Prognostic implications of fibrotic focus (scar) in small peripheral lung cancers. Am J Surg Pathol. 1980;4: 365-73.

7. Noguchi M, Morikawa A, Kawasaki M, Matsuno Y, Yamada T, Hirohashi S, et al. Small adenocarcinoma of the lung. Histologic characteristics and prognosis. Cancer. 1995;75:2844-52.

8. Takashima S, Li F, Maruyama Y, Hasegawa M, Takayama F, Kadoya $\mathrm{M}$, et al. Discrimination of subtypes of small adenocarcinoma in the lung with thin-section CT. Lung Cancer. 2002;36:175-82.

9. van Kuppevelt TH, Robbesom AA, Versteeg EM, Veerkamp JE, van Herwaarden CL, Dekhuijzen PN. Restoration by vacuum inflation of original alveolar dimensions in small human lung specimens. Eur Respir J. 2000;15:771-7.

10. Goto K, Yokose T, Kodama T, Nagai K, Nishiwaki Y, Ando M, et al. Detection of early invasion on the basis of basement membrane destruction in small adenocarcinomas of the lung and its clinical implications. Mod Pathol. 2001;14:1237-45.

11. Yokose T, Suzuki K, Nagai K, Nishiwaki Y, Sasaki S, Ochiai A. Favorable and unfavorable morphological prognostic factors in peripheral adenocarcinoma of the lung $3 \mathrm{~cm}$ or less in diameter. Lung Cancer. 2000;29:179-88. 
12. Narita Y, Nagai K, Yoshida J, Nishimura M, Takahashi K, Kodama $\mathrm{T}$, et al. Clinico-pathological study of c-stage I small $(\mathrm{T}<2$ cm) lung cancer. J Jpn Assoc Chest Surg. 1996;16:46-51 (in Japanese)

13. Miller DL, Rowland CM, Deschamps C, Allen MS, Trastek VF, Pairolero PC. Surgical treatment of non-small cell lung cancer $1 \mathrm{~cm}$ or less in diameter. Ann Thorac Surg. 2002;73:1545-50.

14. Miller JI Jr. Limited resection of bronchogenic carcinoma in the patient with impaired pulmonary function. Ann Thorac Surg. 1993; 56:769-71.

15. Aoki T, Nakata H, Watanabe H, Nakamura K, Kasai T, Hashimoto H, et al. Evolution of peripheral lung adenocarcinomas: CT findings correlated with histology and tumor doubling time. AJR Am J Roentgenol. 2000;174:763-8.

16. Mori M, Chiba R, Takahashi T. Atypical adenomatous hyperplasia of the lung and its differentiation from adenocarcinoma. Characterization of atypical cells by morphometry and multivariate cluster analysis. Cancer. 1993;72:2331-40.

17. Kondo T, Yamada K, Noda K, Nakayama H, Kameda Y. Radiologicprognostic correlation in patients with small pulmonary adenocarcinomas. Lung Cancer. 2002;36:49-57.

18. Hasegawa M, Sone S, Takashima S, Li F, Yang ZG, Maruyama Y, et al. Growth rate of small lung cancers detected on mass CT screening. Br J Radiol. 2000;73:1252-9.

\section{JTCVS On-Line Manuscript Submission and Review}

Please visit http://www.editorialmanager.com/jtcvs/

Effective September 15, 2001, authors and reviewers may submit manuscripts and reviews electronically via Editorial Manager, our new Web-based system with full electronic submission, review, and status update capabilities.

As we move from paper to electronic submissions, the Editorial Office will make proxy submissions of all manuscripts accompanied by a diskette containing the electronic files of the text, tables, and figures. Editors, authors, and reviewers will receive automatic e-mails when significant events occur.

We strongly encourage all authors and reviewers to use Editorial Manager. Although we will continue to accommodate the submission of paper manuscripts for some months, our goal is to be completely electronic within 9 to 12 months.

All individuals currently in our database for whom we have e-mail addresses will receive via e-mail a system-assigned username and password that can be used to log in to the system without prior registration. All those not receiving the e-mail must register the first time they use the system.

As with any broad systemic change, the conversion to the new system will take some time to complete. We ask your patience as we replace our in-office database with the new system. We also encourage you to take advantage of the speed and efficiency that the new system will provide for us all: editor, author, reviewer, and publisher. 